\title{
The 786-0 renal cancer cell-derived exosomes promote angiogenesis by downregulating the expression of hepatocyte cell adhesion molecule
}

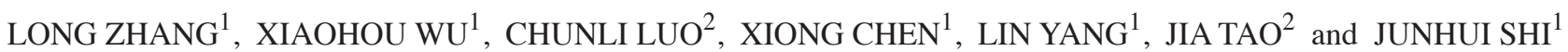 \\ ${ }^{1}$ Department of Urology, The First Affiliated Hospital of Chongqing Medical University; \\ ${ }^{2}$ College of Laboratory Medicine, Key Laboratory of Medical Diagnostics in the Ministry of Education, \\ Chongqing Medical University, Chongqing 400016, P.R. China
}

Received November 30, 2012; Accepted April 25, 2013

DOI: $10.3892 / \mathrm{mmr} .2013 .1458$

\begin{abstract}
The aims of the current study were to determine whether 786-0 renal cancer cell-derived exosomes promote human umbilical vein endothelial cells (HUVECs) to form tubular structures and to uncover the underlying mechanisms associated with this process. Exosomes were extracted and purified using ultrafiltration and sucrose gradient centrifugation and characterized by transmission electron microscopy. Tubular structure formation was observed using the matrigel tubular assay. In addition, an adenovirus vector was used to transfect the hepatocyte cell adhesion molecule (hepaCAM) gene into renal cancer 786- 0 cells. The expression of hepaCAM and vascular endothelial growth factor (VEGF) mRNA and protein was determined by reverse transcription-polymerase chain reaction and western blot analysis, respectively. Tumor cell-derived exosomes were observed to significantly increase tubular formation in HUVECs. Following transfection with the hepaCAM gene, VEGF expression in 786-0 cells was markedly decreased. In HUVECs, exosome treatment increased VEGF mRNA and protein expression, while hepaCAM expression was only decreased at the protein level. In the present study, renal cancer 786-0 cell-derived exosomes significantly promoted angiogenesis via upregulation of VEGF expression in HUVECs, which may be induced by the downregulation of hepaCAM.
\end{abstract}

\section{Introduction}

Renal cancer is a urinary tumor that affects individuals worldwide, accounting for $\sim 3 \%$ of all systemic malignan-

Correspondence to: Professor Xiaohou Wu, Department of Urology, The First Affiliated Hospital of Chongqing Medical University, 1 You Yi Road, Chongqing 400016, P.R. China

E-mail:wuxiaohou80@hotmail.com

Key words: hepatocyte cell adhesion molecule, exosome, vascular endothelial growth factor, renal cancer, angiogenesis, intercellular communication cies (1). Furthermore, it is one of the most common forms of cancer in China. Exosomes are classically defined as membranous vesicles with a diameter of 30-100 $\mathrm{nm}$ and cup-shaped morphology that are secreted by a broad array of cells during physiological and pathological conditions (2). These organelles exert versatile functions due to significant variations in their contents from the originating cell, including a large array of proteins, RNA, mRNA and lipids $(2,3)$. Previous studies reported that extracellular organelles are important mediators of intercellular community (4-9). Tumor cell-derived exosomes are associated with numerous events in cancer pathogenesis and development, including tumor angiogenesis (10).

Beginning at the early phases of the neoplastic process, tumor cells begin to manipulate the host environment to favor their survival and growth (11). Vessels are markedly associated with the pathogenesis and development of tumors and it has been demonstrated that cells at the preneoplastic stage must acquire angiogenic capacity to become malignant cells. Without blood vessels, tumors cannot grow and form metastases (12-14). Vascular endothelial growth factor (VEGF) is the most important factor for the induction and regulation of proliferation of vascular endothelial cells as well as angiogenesis in physiological and pathological conditions. High expression of VEGF has been detected in kidney cancer tissue and serum $(3,15)$. Thus, VEGF is an important target for studies in renal cancer immunity therapy. Hepatocyte cell adhesion molecule (hepaCAM) was previously identified as a novel member of the immunoglobulin super family and was undetectable or expressed at low levels in a number of cancer cells and tissues, including renal cancer (16-18). Therefore, hepaCAM has been hypothesized to be a candidate tumor suppressor gene. In our previous study, hepaCAM was undetectable in transitional cell carcinoma of bladder cell lines T24 and BIU-87, and low hepaCAM levels were found to correlate with increased VEGF levels (19), indicating that hepaCAM is important in suppression of tumor angiogenesis.

Thus, the aim of the current study was to determine whether renal cancer-derived exosomes upregulate VEGF expression via the downregulation of hepaCAM expression, leading to the promotion of angiogenesis. 


\section{Materials and methods}

Cell lines and culture. Human renal cancer cell line, 786-0 and human umbilical vein endothelial cell (HUVEC) line, hy-926, were gifts from the College of Laboratory Medicine (Chongqing Medical University). The cell lines were maintained in RPMI-1640 medium (Gibco-BRL, Shanghai, China) supplemented with $10 \%$ fetal bovine serum (Hyclone Laboratories, Inc., Logan, UT, USA) in a $5 \% \mathrm{CO}_{2}$ humid incubator at $37^{\circ} \mathrm{C}$. Experiments were performed at the cell logarithmic growth phase.

Adenovirus transfection. When cell confluence reached $90 \%$, serum-free medium was exchanged and the adenovirus solution (recombinant adenovirus AdI-EGFP and AdI-hepaCAM) was added to the flask. Complete medum (RPMI-1640 medium supplemented with $10 \%$ fetal bovine serum) was added following $1.5 \mathrm{~h}$ and protein from each group was extracted following 72-h incubation.

Extraction and identification of exosomes. Supernatants of cultured 786-0 cells were collected and subsequently centrifuged at $4^{\circ} \mathrm{C}$ at $300 \mathrm{x} \mathrm{g}$ for $10 \mathrm{~min}, 800 \mathrm{x} \mathrm{g}$ for $30 \mathrm{~min}$ and $10,000 \mathrm{x} g$ for $30 \mathrm{~min}$ to deposit cells and debris. Supernatants were concentrated by ultrafiltration using a $100 \mathrm{kDa} \mathrm{MWCO}$ Centriplus centrifugal ultrafiltration tube (Millipore, Billerica, MA, USA) at 1,000 x g for $30 \mathrm{~min}$. Remaining supernatants were concentrated and subjected to ultracentrifugation in a centrifugal ultrafiltration tube containing $30 \%$ sucrose in heavy water (Tenglong Weibo Technology, Qingdao, China) at $100,000 \times \mathrm{g}$ for $1 \mathrm{~h}$ at $4^{\circ} \mathrm{C}$. The sucrose solution was collected and diluted with phosphate-buffered saline, followed by concentration using an additional $100 \mathrm{kDa}$ MWCO Centriplus centrifugal ultrafiltration tube at $1,000 \mathrm{x} \mathrm{g}$ for $30 \mathrm{~min}$. Finally, the remaining exosome-containing solution was collected, filtered through a $0.22 \mu \mathrm{m}$ filter, aliquoted and stored at $-80^{\circ} \mathrm{C}$. Exosomes were characterized by transmission electron microscopy.

Exosome suspension $(20 \mu 1)$ was dropped on the copper-net and the sample was dried using filter paper $1 \mathrm{~min}$ later. Subsequently, the sample was negatively stained by $2 \%$ Salkowski's solution for $1 \mathrm{~min}$ and dried using incandescent lights for $10 \mathrm{~min}$. The sample was then observed and images were captured using transmission electron microscopy.

Matrigel tubular assay. Matrigel (BD Biosciences, Franklin Lakes, NJ, USA) was thawed at $4^{\circ} \mathrm{C}$, applied to a 24 -well plate and incubated at $37^{\circ} \mathrm{C}$ for $12 \mathrm{~h}$ to allow solidification. Then, hy926 cells were seeded onto the matrigel at $1 \times 10^{5}$ cells/well with or without renal cell-derived exosomes. Following incubaton for $72 \mathrm{~h}$, tubular formation of the cells was observed and images were captured. The assay was performed in 5 wells/group.

Reverse transcription-polymerase chain reaction (RT-PCR) analysis. Total RNA was isolated from cells using TRIzol (Takara Bio, Inc., Shiga, Japan) and semi-quantitative RT-PCR was performed using the Two-step RT-PCR kit (Takara Bio, Inc.) following the manufacturer's instructions. Primers were designed using Primer Premier 5.0 (Premier Biosoft, Palo
Alto, CA, USA) and gene primer specificity was confirmed by BLAST search using the GeneBank database. The primers used were: hepaCAM, forward: 5'-TAC TGT AGA TGT GCC CAT TTC G-3' and reverse: 5'-CTT CTG GTT TCA GGC GGT C-3'; VEGF, forward: 5'-GTC CAA CTT CTG GGC TGT TCT-3' and reverse: 5'-ACC ACT TCG TGA TGA TTC TGC-3'; and $\beta$-actin (loading control), forward: 5'-TGA CGT GGA CAT CCG CAA AG-3' and reverse: 5'-CTG GAA GGT GGA CAG CGA GG-3'. Amplified hepaCAM, VEGF and $\beta$-actin fragments were 461, 497 and 205-bp in length, respectively. Total RNA was reverse transcribed and RT-PCR was performed using $1 \mu \mathrm{l} \mathrm{cDNA}$ and primers for relevant genes under the following optimized conditions: predenaturation, $95^{\circ} \mathrm{C}$ for $5 \mathrm{~min} ; 35$ cycles of denaturation at $95^{\circ} \mathrm{C}$ for $30 \mathrm{sec}$, annealing at $56^{\circ} \mathrm{C}, 59^{\circ} \mathrm{C}$ or $56^{\circ} \mathrm{C}$ for $30 \mathrm{sec}$ and extension at $72^{\circ} \mathrm{C}$ for $1 \mathrm{~min}$; and final extension, $72^{\circ} \mathrm{C}$ for $5 \mathrm{~min}$. Products were analyzed by $1.5 \%$ gel electrophoresis using a Bio-Rad imaging plate (Bio-Rad, Hercules, CA, USA).

Western blot analysis. Cells were solubilized in lysis buffer (Beyotime Institute of Biotechnology, Jiangsu, China) containing $1 \mu \mathrm{l}$ phenylmethanesulfonyl fluoride and then centrifuged at $4^{\circ} \mathrm{C}$ at $13200 \mathrm{x}$ g for $5 \mathrm{~min}$ to obtain the supernatant. The concentration was determined by BCA method. Proteins were separated using $10 \%$ sodium dodecyl sulfate-polyacrylamide gel electrophoresis and the protein bands were transferred to polyvinylidene fluoride membranes (Amersham Pharmacia Biotech, Amersham, UK). Membranes were blocked in $5 \%$ skimmed milk for $2 \mathrm{~h}$ and incubated with anti-hepaCAM (Wuhan Sanying Biotechnology Inc., Wuhan, China), anti-VEGF and anti- $\beta$-actin (Wuhan Boster Biological Technology, Ltd., Wuhan, China) antibodies overnight at $4^{\circ} \mathrm{C}$. Following three 10 min washes with TBST, membranes were incubated with HRP-conjugated secondary antibody for $1.5 \mathrm{~h}$. Membranes were washed again (three 10 min washes with TBST) and the immunoreactive bands were detected using an enhanced chemoluminescence kit (Beyotime Institute of Biotechnology, China) in the dark. $\beta$-actin was used as an internal control. The intensity of the protein bands was quantified using Quantity-One software (Bio-Rad).

Statistical analysis. Statistical differences between the groups were analyzed using the Kruskal-Wallis test. Data are presented as mean $\pm \mathrm{SD}$. $\mathrm{P}<0.05$ was considered to indicate a statistically significant difference.

\section{Results}

Morphological identification of exosomes. TEM analysis of exosomes indicated that typical characteristics of a cup-shaped or saucer-like structure with a size ranging from 30-100 nm in diameter (Fig. 1).

In vitro tube formation of exosome-treated HUVECs is markedly increased by treatment with exosomes. HUVECs formed tubular structures in the matrigel and the effect was examined $72 \mathrm{~h}$ following treatment. Compared with the control group, cells treated with 50 or $150 \mu \mathrm{g} / \mathrm{ml}$ exosomes exhibited a marked increase in the formation of tubular structures (both $\mathrm{P}<0.01$; Fig. 2). 


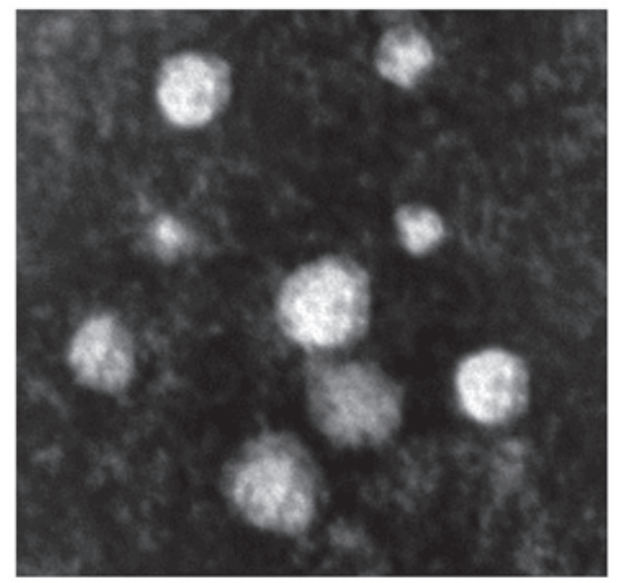

Figure 1.786-0 cell-derived exosomes visualized under electron microscopy (original magnification, $\mathrm{x} 50,000$ ).

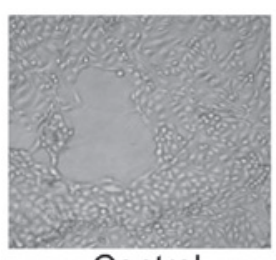

Control

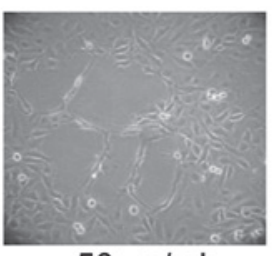

$50 \mu \mathrm{g} / \mathrm{ml}$

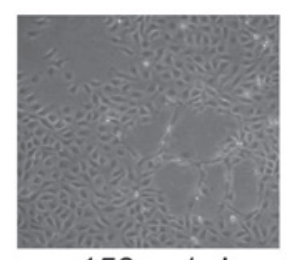

$150 \mu \mathrm{g} / \mathrm{ml}$

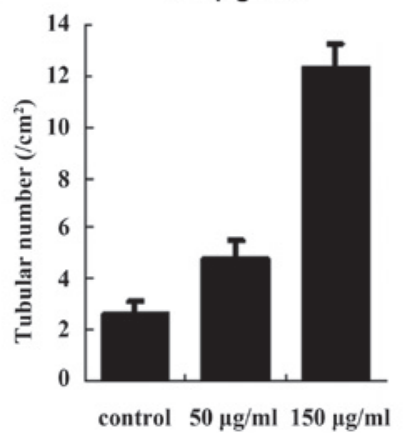

Figure 2. Tube formation of exosome-treated HUVECs was markedly increased in vitro. Compared with the control, groups treated with 50 or $150 \mu \mathrm{g} / \mathrm{ml}$ exosomes exhibited increased formation of tubular structures (magnification, x100). HUVECs, human umbilical vein endothelial cells.

Re-expression of hepaCAM in 786-0 cells downregulates VEGF protein expression. hepaCAM expression was not detected in 786-0 cells and high VEGF expression was observed. Following transfection with AdI-hepaCAM, cells revealed increasing hepaCAM expression and decreasing VEGF expression compared with the AdI-EGFP group (both $\mathrm{P}<0.01$; Fig. 3).

hepaCAM and VEGF expression in HUVECs following treatment with exosomes. HUVECs expressed high levels of hepaCAM mRNA and a low expression of VEGF mRNA. Following treatment with renal cancer cell-derived exosomes, VEGF mRNA expression was found to be markedly decreased compared with the control group (50 and $150 \mu \mathrm{g} / \mathrm{ml}$, both $\mathrm{P}<0.01$; Fig. 4). Expression of hepaCAM mRNA was not found to be statistically significant compared with the control (50 and $150 \mu \mathrm{g} / \mathrm{ml}$, both $\mathrm{P}<0.01$; Fig. 5). Western blot analysis revealed that, compared with the control group, VEGF protein expression was markedly increased (50 and

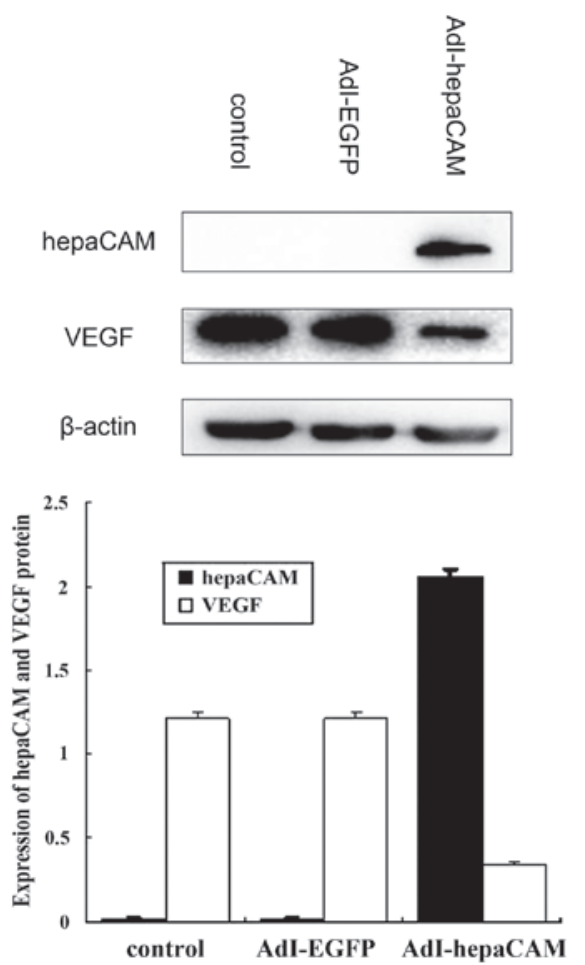

Figure 3. Re-expression of hepaCAM in 786-0 cells downregulated VEGF protein expression. hepaCAM gene was successfully transfected into 786-0 cells using recombinant adenovirus vehicles and VEGF expression was significantly decreased under hepaCAM re-expression. hepaCAM, hepatocyte cell adhesion molecule; VEGF, vascular endothelial growth factor.
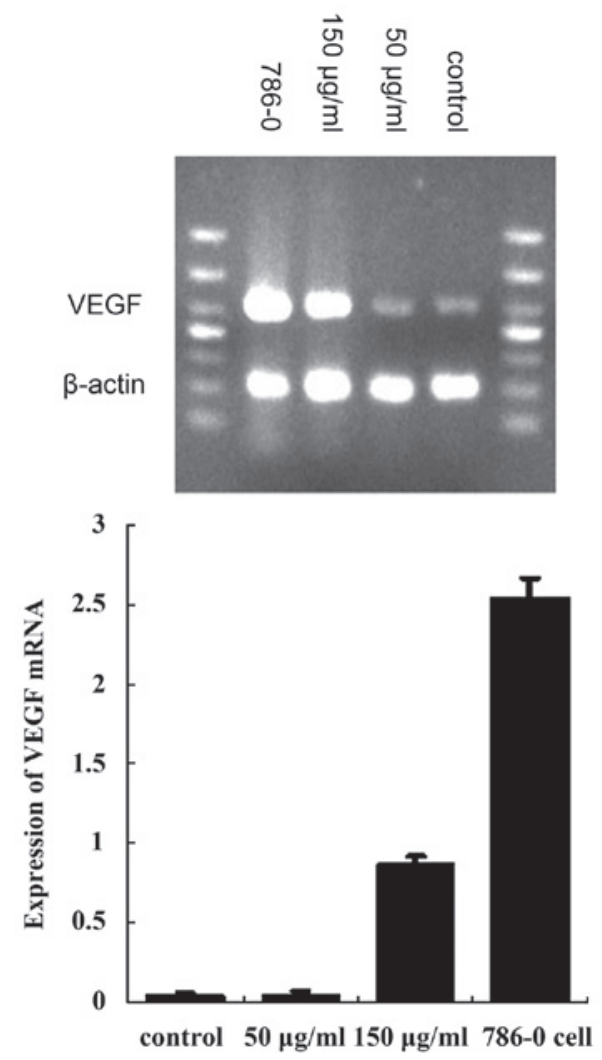

Figure 4. VEGF mRNA expression following exosome treatment. Amplified VEGF and $\beta$-actin fragments were 497 and 205-bp in length, respectively. RT-PCR revealed that $150 \mu \mathrm{g} / \mathrm{ml}$ exosome treatment significantly increased VEGF expression. VEGF, vascular endothelial growth factor; RT-PCR, reverse transcription-polymerase chain reaction. 


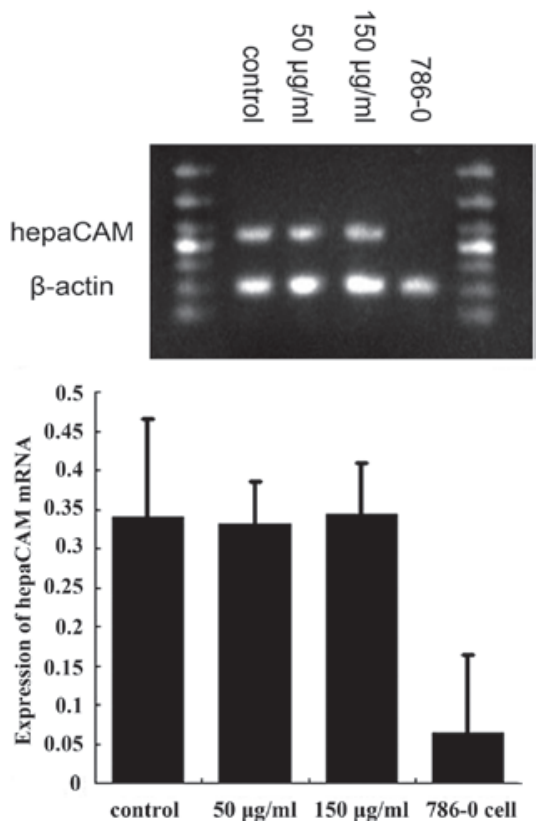

Figure 5. hepaCAM mRNA expression following exosome treatment. Amplified hepaCAM and $\beta$-actin fragments were 461 and 205-bp in length, respectively. RT-PCR demonstrated that exosome treatment had no effect on hepaCAM mRNA expression. hepaCAM, hepatocyte cell adhesion molecule; RT-PCR, reverse transcription-polymerase chain reaction.

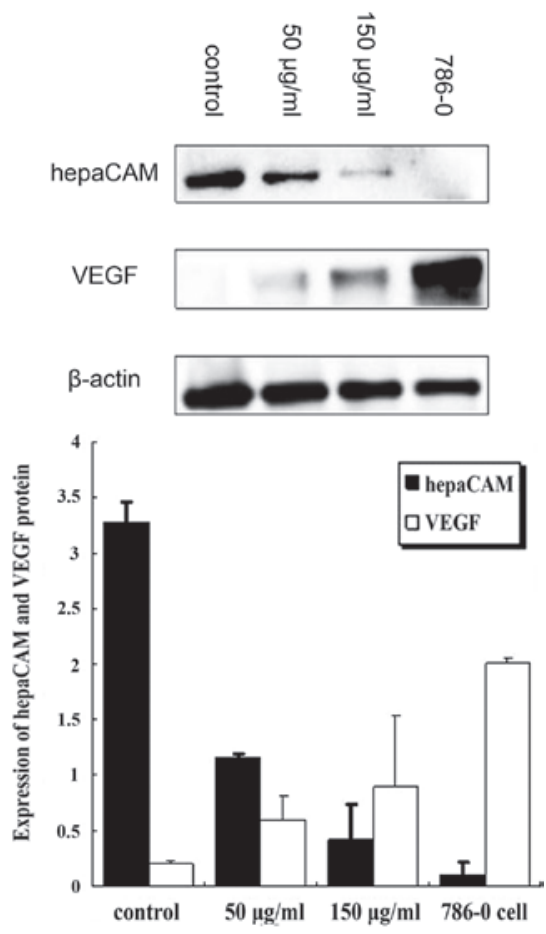

Figure 6. hepaCAM and VEGF protein expression following 50 or $150 \mu \mathrm{g} / \mathrm{ml}$ exosome treatment. Groups revealed a reduced expression of hepaCAM and an increased expression of VEGF compared with control. hepaCAM, hepatocyte cell adhesion molecule; VEGF, vascular endothelial growth factor.

$150 \mu \mathrm{g} / \mathrm{ml}$, both $\mathrm{P}<0.01)$ and hepaCAM protein expression was significantly decreased (50 and $150 \mu \mathrm{g} / \mathrm{ml}$, both $\mathrm{P}<0.01)$. Levels of VEGF were found to inversely correlate with that of hepaCAM (Fig. 6).

\section{Discussion}

Extensive studies on tumor cell-derived exosomes and their roles in intercellular communication in the tumor microenvironment have been performed $(6,8,9,20-24)$. The tumor cell-derived exosome is known to manipulate the surrounding microenvironment to promote angiogenesis, invasion and metastasis, as well as escape immune surveillance (10). In the present study, exosomes secreted from the human renal cancer cell line, 786-0, were found to facilitate tubular formation via regulation of hepaCAM and VEGF expression of HUVECs.

Consistent with a number of previous studies (10,25-27), 786-0 cell-derived exosomes were observed to increase the formation of tubular stuctures in HUVECs compared with the control group. However, the underlying molecular mechanism of this effect remains unclear. Al-Nedawi et al revealed that exosomes transfer the oncogenic form of EGFR, EGFRvIII, from glioblastoma multiforme cells to endothelial cells (24), resulting in EGFRvIII-driven endothelial expression of autocrine VEGF. In the present study, expression of VEGF, an important factor in angiogenesis in physiological and pathological conditions, was upregulated in HUVECs at the mRNA and protein levels following treatment with cancer cell-derived exosomes, while hepaCAM protein levels decreased. In addition, re-expression of hepaCAM markedly reduced the expression of VEGF in 786-0 cells, which was consistent with our previous study (19). Induction of angiogenesis by VEGF has been found to be facilitated by the downregulation of expression (28) or decreasing stability of p53 (29) and is inhibited when p53 protein is upregulated (30). Re-expression of hepaCAM elevates p53 protein levels, while the knockdown of endogenous p53 expression via small-interfering RNA alleviates the proliferation inhibition of hepaCAM (17). In the current study, decreased hepaCAM partly induced increased levels of VEGF in HUVECs and the p53 signaling pathway was hypothesized to be be involved in this process.

In addition, no significant change in hepaCAM mRNA expression was identified and the lower protein level may be associated with post-transcriptional regulation. The specific mechanism by which hepaCAM protein is reduced and the associated signaling pathway requires further analysis. In a previous study, Zhang et al analyzed human breast carcinoma MCF7 cells, identifying a cleaved form of hepaCAM associated with the proteasome, calpain-1 and cathepsin B (18). Tumor cell-generated exosomes may directly modify adhesion molecules following transfer of these enzymes from parent to recipient cells. By contrast, specific immunoglobulin superfamily adhesion molecules, including ICAM-1, from activated endothelial cells are shed in soluble form, which may promote angiogenesis (31). Exosomes may also affect hepaCAM expression by activating the endothelial cells and shedding them from the membrane. In addition, the signaling pathways associated with exosome regulation of hepaCAM and VEGF remain unknown and require additional analysis.

In the present study, renal cancer 786-0 cell-derived exosomes significantly promoted angiogenesis via upregulation of VEGF expression in HUVECs, which may be induced by the downregulation of hepaCAM. 


\section{Acknowledgements}

The authors would like to thank Dr Shali Shen for the kind support throughout the study and Professor Weixue Tang for providing technical guidance.

\section{References}

1. Jemal A, Siegel R, Xu J and Ward E: Cancer statistics, 2010. CA Cancer J Clin 60: 277-300, 2010.

2. Keller S, Sanderson MP, Stoeck A and Altevogt P: Exosomes: from biogenesis and secretion to biological function. Immunol Lett 107: 102-108, 2006.

3. Iero M, Valenti R, Huber V, et al: Tumour-released exosomes and their implications in cancer immunity. Cell Death Differ 15: 80-88, 2008

4. Ratajczak J, Wysoczynski M, Hayek F, Janowska-Wieczorek A and Ratajczak MZ: Membrane-derived microvesicles: important and underappreciated mediators of cell-to-cell communication. Leukemia 20: 1487-1495, 2006.

5. Quesenberry PJ and Aliotta JM: The paradoxical dynamism of marrow stem cells: considerations of stem cells, niches and microvesicles. Stem Cell Rev 4: 137-147, 2008.

6. Cocucci E, Racchetti G and Meldolesi J: Shedding microvesicles: artefacts no more. Trends Cell Biol 19: 43-51, 2009.

7. Théry C: Exosomes: secreted vesicles and intercellular communications. F1000 Biol Rep 3: 15, 2011.

8. Mathivanan S, Ji H and Simpson RJ: Exosomes: extracellular organelles important in intercellular communication. J Proteomics 73: 1907-1920, 2010.

9. Fevrier B and Raposo G: Exosomes: endosomal-derived vesicles shipping extracellular messages. Curr Opin Cell Biol 16: 415-421, 2004.

10. Marleau AM, Chen CS, Joyce JA and Tullis RH: Exosome removal as a therapeutic adjuvant in cancer. J Transl Med 10: $134,2012$.

11. Lin WW and Karin M: A cytokine-mediated link between innate immunity, inflammation and cancer. J Clin Invest 117: 1175-1183, 2007.

12. Goth MI, Hubina E, Raptis S, Nagy GM and Toth BE: Physiological and pathological angiogenesis in the endocrine system. Microsc Res Tech 60: 98-106, 2003.

13. Pircher A, Medinger M and Drevs J: Liver cancer: Targeted future options. World J Hepatol 3: 38-44, 2011.

14. Carmeliet P and Jain RK: Angiogenesis in cancer and other diseases. Nature 407: 249-257, 2000.

15. Yang $\mathrm{R}$, Zhang $\mathrm{H}$ and Zhu L: Inhibitory effect of resveratrol on the expression of the VEGF gene and proliferation in renal cancer cells. Mol Med Rep 4: 981-983, 2011.

16. He Y, Wu X, Luo C, Wang L and Lin J: Functional significance of the hepaCAM gene in bladder cancer. BMC Cancer 10: 83 , 2010.
17. Moh MC, Zhang T, Lee LH and Shen S: Expression of hepaCAM is downregulated in cancers and induces senescence-like growth arrest via a p53/p21-dependent pathway in human breast cancer cells. Carcinogenesis 29: 2298-2305, 2008.

18. Zhang T, Moh MC, Lee LH and Shen S: The immunoglobulin-like cell adhesion molecule hepaCAM is cleaved in the human breast carcinoma MCF7 cells. Int J Oncol 37: 155-165, 2010.

19. Yang S, Wu X, Luo C, Pan C and Pu J: Expression and clinical significance of hepaCAM and VEGF in urothelial carcinoma. World J Urol 28: 473-478, 2010.

20. Belting $\mathbf{M}$ and Wittrup A: Nanotubes, exosomes and nucleic acid-binding peptides provide novel mechanisms of intercellular communication in eukaryotic cells: implications in health and disease. J Cell Biol 183: 1187-1191, 2008.

21. Al-Nedawi K, Meehan B and Rak J: Microvesicles: messengers and mediators of tumor progression. Cell Cycle 8: 2014-2018, 2009.

22. Skog J, Würdinger T, van Rijn S, et al: Glioblastoma microvesicles transport RNA and proteins that promote tumour growth and provide diagnostic biomarkers. Nat Cell Biol 10: 1470-1476, 2008

23. Al-Nedawi K, Meehan B, Micallef J, et al: Intercellular transfer of the oncogenic receptor EGFRvIII by microvesicles derived from tumour cells. Nat Cell Biol 10: 619-624, 2008.

24. Al-Nedawi K, Meehan B, Kerbel RS, Allison AC and Rak J: Endothelial expression of autocrine VEGF upon the uptake of tumor-derived microvesicles containing oncogenic EGFR. Proc Natl Acad Sci USA 106: 3794-3799, 2009.

25. Corrado C, Flugy AM, Taverna S, et al: Carboxyamidotriazoleorotate inhibits the growth of imatinib-resistant chronic myeloid leukaemia cells and modulates exosomes-stimulated angiogenesis. PLoS One 7: e42310, 2012.

26. Grange C, Tapparo M, Collino F, et al: Microvesicles released from human renal cancer stem cells stimulate angiogenesis and formation of lung premetastatic niche. Cancer Res 71: 5346-5356, 2011.

27. Martinez MC and Andriantsitohaina R: Microparticles in angiogenesis: therapeutic potential. Circ Res 109: 110-119, 2011.

28. Song H, Yin D and Liu Z: GDF-15 promotes angiogenesis through modulating p53/HIF-1alpha signaling pathway in hypoxic human umbilical vein endothelial cells. Mol Biol Rep 39: 4017-4022, 2012.

29. Ma J, Xue Y, Cui W, et al: Ras homolog gene family, member A promotes p53 degradation and vascular endothelial growth factor-dependent angiogenesis through an interaction with murine double minute 2 under hypoxic conditions. Cancer 118: 4105-4116, 2012.

30. Ling Y, Chen Y, Chen P, et al: Baicalein potently suppresses angiogenesis induced by vascular endothelial growth factor through the $\mathrm{p} 53 / \mathrm{Rb}$ signaling pathway leading to $\mathrm{G} 1 / \mathrm{S}$ cell cycle arrest. Exp Biol Med (Maywood) 236: 851-858, 2011.

31. Reinmuth N, Thomas M, Meister M, Schnabel PA and Kreuter M: Current data on predictive markers for anti-angiogenic therapy in thoracic tumours. Eur Respir J 36: 915-924, 2010. 